\title{
MAXIMAL IDEALS IN THE BURNSIDE RING OF A COMPACT LIE GROUP
}

\author{
STEFAN BAUER AND J. P. MAY
}

(Communicated by Haynes R. Miller)

\begin{abstract}
A new criterion is found for deciding whether or not two maximal ideals in the Burnside ring of a compact Lie group coincide. One consequence is that certain algebraic and topological localizations in equivariant stable homotopy theory are naturally isomorphic.
\end{abstract}

Let $G$ be a compact Lie group and $H$ a (closed) subgroup. For a prime $p$, let $q(H, p)$ be the kernel of the homomorphism $A(G) \rightarrow Z_{p}$ obtained by sending the equivalence class of a finite $G-\mathrm{CW}$ complex $X$ to the $\bmod p$ reduction of the Euler characteristic $\chi\left(X^{H}\right)$. All maximal ideals of the Burnside ring $A(G)$ are of this general form. Our main goal is to obtain a satisfactory criterion for determining when $q(J, p)=q(H, p)$. The reader is referred to [1, 5.7 or $2, \mathrm{~V}, \S 3]$ for background and earlier results. We shall obtain the following result as a consequence of our criterion.

THEOREM 1. If $H \subset J \subset K$ and $q(H, p)=q(K, p)$, then $q(J, p)=q(K, p)$.

This gives a positive answer to a question raised in [2, V.3.8]. As explained in detail in [2, V.8.9], the theorem has the following consequence in equivariant stable homotopy theory. Let $W H=N H / H$.

THEOREM 2. Let $q=q(K, p)$, where $|W K|$ is finite and prime to $p$, and let

$$
\begin{aligned}
\mathcal{E} & =\{H \mid q(J, p) \neq q \text { for all } J \subset H\}, \\
\mathcal{F}^{\prime} & =\{H \mid(H) \leq(K)\} \text { and } \mathcal{F}=\mathcal{E} \cap \mathcal{F}^{\prime} .
\end{aligned}
$$

Then, for finite $G-C W$ spectra $X$ and general $G$-spectra $Y$, the algebraic localization $[X, Y]_{q}^{G}$ is naturally isomorphic to the topological localization $\left[X, E\left(\mathcal{F}^{\prime}, \mathcal{F}\right) \wedge Y\right]_{(p)}^{G}$.

Here $E\left(\mathcal{F}^{\prime}, \mathcal{F}\right)$ is a based $G$-CW complex characterized up to $G$-equivalence by the requirement that $E\left(\mathcal{F}^{\prime}, \mathcal{F}\right)^{H}$ be equivalent to $S^{0}$ if $H \in \mathcal{F}^{\prime}-\mathcal{F}$ and be contractible otherwise (see e.g. [2, V, $\S 7]$ ). The importance of Theorem 2 is that $[X, Y]_{(p)}^{G}$ can be computed in terms of the various algebraic localizations $[X, Y]_{q}^{G}$ (by [2, V.5.5]). The relevance of Theorem 1 is that it implies

$$
\mathcal{F}^{\prime}-\mathcal{F}=\{H \mid q(H, p)=q(K, p)\} .
$$

To see this, recall that, for any maximal ideal $q$ of residual characteristic $p$, there is one and, up to conjugacy, only one $K$ with $|W K|$ finite and prime to $p$ such that

Received by the editors October 6, 1986.

1980 Mathematics Subject Classification (1985 Revision). Primary 57S99.

Key words and phrases. Burnside ring, compact Lie group, equivariant stable homotopy, localization. 
$q=q(K, p)$; moreover, this $K$ is maximal in $\{H \mid q(H, p)=q\}$ (see $[\mathbf{1}, 5.7 .2$ or $\mathbf{2}$, V.3.1]). For a given $H$, we let $H^{p}$ denote a subgroup of $G$ such that $q(H, p)=$ $q\left(H^{p}, p\right)$ and $\left|W H^{p}\right|$ is finite and prime to $p$.

If $G$ is finite and $H_{p}$ is the smallest normal subgroup of $H$ such that $H / H_{p}$ is a $p$-group, then $q(H, p)=q(J, p)$ if and only if $H_{p}$ is conjugate to $J_{p}$. We wish to generalize this criterion to the compact Lie case. Say that a group is $p$-perfect if it admits no nontrivial quotient $p$-groups. Then $H_{p}$ above can also be characterized as the maximal $p$-perfect subgroup of $H$. In the compact Lie case, we let $H_{p}^{\prime}$ denote the maximal $p$-perfect subgroup of $H$. It can be constructed explicitly as the inverse image in $H$ of $\left(H / H_{0}\right)_{p} \subset H / H_{0}$, where $H_{0}$ is the component of the identity element. We then define $H_{p} \subset N H_{p}^{\prime}$ to be the inverse image of a maximal torus in $W H_{p}^{\prime} . H_{p}$ is still $p$-perfect, but now $W H_{p}$ is finite [1, 5.7.5 or 2, V.3.3]. Note that $q(H, p)=q\left(H_{p}^{\prime}, p\right)=q\left(H_{p}, p\right)$ since $H / H_{p}^{\prime}$ is a $p$-group and $H_{p} / H_{p}^{\prime}$ is a torus $[1,5.7 .1$ or 2, V.3.6]. The following is our main result. We are indebted to the referee for its proper formulation.

THEOREM 3. Let $q=q(K, p)$, where $|W K|$ is finite and prime to $p$, and let $L=K_{p}$.

(i) $L=K_{p}^{\prime}$; that is, $L$ is the maximal p-perfect subgroup of $K$; moreover, $L$ is the unique normal p-perfect subgroup of $K$ whose quotient is a finite p-group.

(ii) $L$ is maximal in $\{H \mid q(H, p)=q$ and $H$ is p-perfect $\}$; up to conjugacy, this property uniquely characterizes $L$.

(iii) If $H \subset G$, then $q(H, p)=q$ if and only if $H_{p}$ is conjugate to $L$.

(iv) If $H \subset L$ is $p$-perfect and $q(H, p)=q$, then $H T=L$, where $T$ is the component of the identity element of the center of $L$.

Part (iii) can be restated as follows.

COROLlaRY 4. $q(H, p)=q(J, p)$ if and only if $H_{p}$ is conjugate to $J_{p}$.

Parts (i) and (iv) imply Theorem 1.

ProOF OF THEOREM 1. We are given $H \subset J \subset K$ and $q(H, p)=q(K, p)$. Expanding $K$ if necessary, we may assume that $|W K|$ is finite and prime to $p$. We have $H_{p}^{\prime} \subset J_{p}^{\prime} \subset K_{p}^{\prime}$ since passage to maximal $p$-perfect subgroups preserves inclusions. By (i), $K_{p}^{\prime}=L$. By (iv), $H_{p}^{\prime} T=L$. Therefore $J_{p}^{\prime} T=L$. Since $J_{p}^{\prime} T / J_{p}^{\prime}$ is a torus,

$$
q(J, p)=q\left(J_{p}^{\prime}, p\right)=q\left(J_{p}^{\prime} T, p\right)=q(K, p) .
$$

The proof of Theorem 3 depends on the following observation.

Proposition 5. If $H$ is $p$-perfect and $W H$ is finite, then, up to conjugation, $H$ is a normal subgroup of $H^{p}$ with quotient a finite p-group.

PROOF. The argument is identical to the proof of $[\mathbf{1}, 5.7 .8$ or 2, V.3.6(iii)], which give the same conclusion under the stronger hypothesis that $\left|H / H_{0}\right| \not \equiv 0$ $\bmod p$ rather than that $H$ is $p$-perfect.

ProOF OF THEOREM 3. (i) By the proposition, $L$ is subconjugate to $K$. Since $K / K_{p}^{\prime}$ is finite and $L / K_{p}^{\prime}$ is a torus, this implies $L=K_{p}^{\prime}$. By the maximality of $K_{p}^{\prime}$, if $H \subset K$ is $p$-perfect, then $H \subset \overline{\text { L }}$. If, further, $H \triangleleft K$ and $K / H$ is a $p$-group, then $H=L$ since $L$ is $p$-perfect. 
(ii) Since $K$ is maximal in $\{H \mid q(H, p)=q\}$ and is characterized up to conjugacy by this property, (ii) follows immediately from (i).

(iii) Since $q(H, p)=q\left(H_{p}, p\right)$, sufficiency is clear. Applying the proposition to $H_{p}$ and then applying part (i) to $H^{p}$, we see that $H_{p}$ is conjugate to $\left(H^{p}\right)_{p}$. This implies necessity.

(iv) Since $H$ is $p$-perfect and $H \triangleleft H T$ with quotient a torus, the construction of $H_{p}$ shows that we may assume $H T \subset H_{p}$. Since $H_{p} / H$ is a torus, $H T \triangleleft H_{p}$ and $H_{p} / H T$ is a torus. As a compact subgroup of the discrete group of automorphisms modulo inner automorphisms of $H T, H_{p} / S H T$ is finite and thus trivial, where $S$ is the centralizer of $H T$ in $H_{p}$. Clearly $S$ contains $T$ and is the center of $H_{p}$. Since $q(H, p)=q, H_{p}$ is conjugate to $L$ by part (iii). Therefore the identity component of $S$ is isomorphic to, and thus equal to, $T$. It follows that $H_{p}=H T \subset L$ and hence $H_{p}=H T=L$.

\section{REFERENCES}

1. $\mathrm{T}$. tom Dieck, Transformation groups and representation theory, Lecture Notes in Math., vol. 766, Springer-Verlag, Berlin and New York, 1979.

2. L. G. Lewis, Jr., J. P. May, and M. Steinberger (with contributions by J. E. McClure), Equivariant stable homotopy theory, Lecture Notes in Math., vol. 1213, Springer-Verlag, Berlin and New York, 1986.

Department of Mathematics, University of Chicago, Chicago, illinois 60637 (Current address of J. P. May)

Current address (Stefan Bauer): Sonderforschungsbereich 170, "Geometrie und Analysis", Mathematisches Institut, Bunsenstrasse 3-5, D-3400 Göttingen, Federal Republic of Germany 